\section{Company Name}

4pi Analysis, Inc.

Abeam Technologies

Advanced MicroBeam, Inc.

Advanced Microscopy Techniques Corp.

Allied High Tech Products, Inc.

Applied Image, Inc.

ASM International

Attocube Systems AG

Biophotonics International

Bruker AXS Microanalysis

Buehler LTD

Cambridge University Press

CAMECA Instruments, Inc.

Carestream Molecular Imaging

Carl Zeiss SMT, Inc.

Chroma Technology Corp.

Delaware Diamond Knives, Inc.

Denton Vacuum, Inc.

DiATOME U.S.

Direct Electron, LP

Dune Sciences, LLC

Duniway Stockroom Corp.

DVC Company

e2v scientific instruments ltd.

EDAX, Inc.

Electron Microscopy Sciences

Energy Beam Sciences, Inc.

Ernest F. Fullam, Inc.

ETS-Lindgren

Evex Analytical

FEI Company

Fischione Instruments

Gatan, Inc.

Hamamatsu Corp.

Hirox-USA, Inc.

Hitachi High Technologies America, Inc.

HORIBA Jobin Yvon, Inc.

HREM Research, Inc.

Hummingbird Scientific

Hysitron, Inc.

Intellection Corp.

International Centre for Diffraction Data (ICDD)

Ionizing Radiation Imaging Systems (IRIS)

IXRF Systems, Inc.

JENOPTIK GmbH

JEOL USA, Inc.

John Wiley \& Sons, Inc.

K E Developments Ltd
Booth \#

1022

417

604

704

613

710

331

823

216

1109

506

828

212

415

804

606

713

927

811

420

335

715

612

614

1122

813

822

619

220

816

528

424

1130

522

1423

1329

311

1325

837

714

1319

623

736

303

712

515, 1027

316

822
Company Name

Ladd Research

Leco Corporation

Leica Microsystems, Inc.

Luxel

The McCrone Group, Inc.

MSA Megabooth

MTS Nano Instruments

Nanofactory Instruments $\mathrm{AB}$

Nascatec GmbH

Nikon Instruments, Inc.

Norsam Technologies, Inc.

Novelx, Inc.

Olympus Industrial America

Olympus America, Inc.

Olympus Soft Imaging Solutions

Omniprobe, Inc.

Oxford Instruments America, Inc.

Parallax Research

Physical Electronics

PNSensor GmbH

Probe Software, Inc.

Protochips, Inc.

PulseTor LLC

Quorum Technologies, Ltd.

Rap-ID US

Renishaw, Inc.

RMC Products Boeckeler Instruments, Inc.

Scientific Instruments \& Applications, Inc.

Sela USA, Inc.

SII NanoTechnology USA, Inc

Siskiyou Corporation

Smart Imaging Technologies

Smiths Detection

South Bay Technology, Inc.

SPI Supplies

Technical Manufacturing Corporation

Technotrade International, Inc.

Ted Pella, Inc.

Tescan USA, Inc.

Thermo Fisher Scientific

Tousimis

TVIPS GmbH

Booth \#

504

1426

430

508

827

130

916

208

520

835

333

607

304

304

304

922

620

929

711

419

318

610

329

822

437

336

603

621

722

126

418

709

416

819

803

510

708

703

330

834

738

224

728

Vibration Engineering Consultants $\quad 608$

Well Diamond Wire Saws, Inc. 320

WITec Instruments Corp.

XEI Scientific, Inc.

Xradia, Inc. 


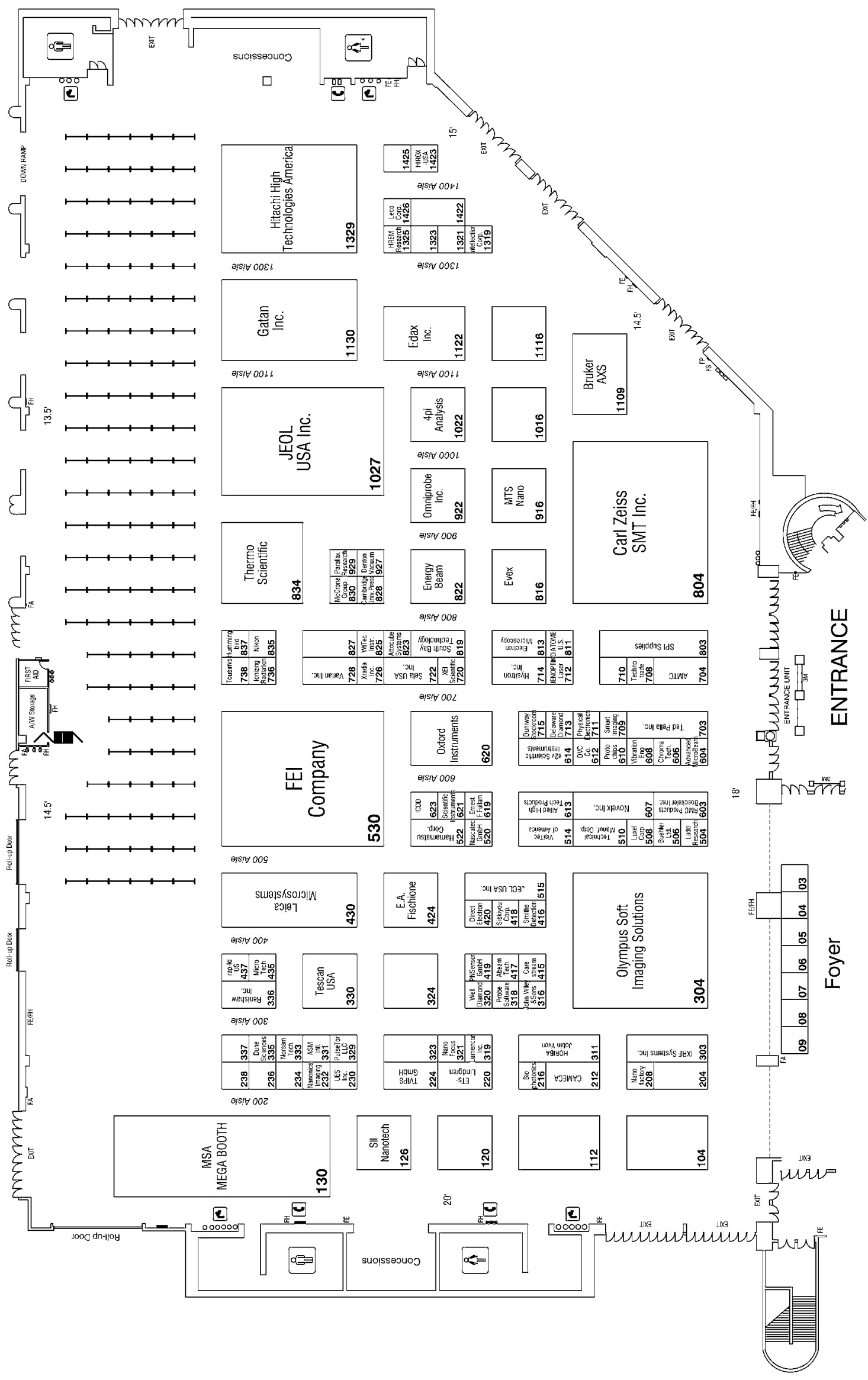

